\title{
Age-of-onset of hypertension vs. a single measurement of systolic blood pressure in a combined linkage and segregation analysis E Warwick Daw*1,2, Xiaoming Liu ${ }^{2}$ and Chih-Chieh Wu ${ }^{1}$
}

\author{
Address: ${ }^{1}$ Department of Epidemiology, University of Texas MD Anderson Cancer Center, 1515 Holcombe Boulevard., Houston, Texas USA and \\ 2Program in Human and Molecular Genetics, University of Texas Graduate School of Biomedical Sciences, Houston, Texas USA \\ Email: E Warwick Daw* - warwick@request.mdacc.tmc.edu; Xiaoming Liu - Xiaoming.Liu@uth.tmc.edu; Chih- \\ ChiehWu - ccwu@request.mdacc.tmc.edu \\ * Corresponding author
}

from Genetic Analysis Workshop 13: Analysis of Longitudinal Family Data for Complex Diseases and Related Risk Factors New Orleans Marriott Hotel, New Orleans, LA, USA, November I I-14, 2002

Published: 31 December 2003

BMC Genetics 2003, 4(Suppl I):S80

This article is available from: http://www.biomedcentral.com/|47I-2I56/4/sI/S80

\begin{abstract}
Background: Often, multiple measures of a trait are available in a genetic linkage analysis. We compare Monte Carlo Markov chain analysis of two very different measures of hypertension in the simulated Genetic Analysis Workshop 13 data to examine how choice of measure affects the results. The measures selected were age-of-onset of hypertension and systolic blood pressure at first visit.

Results: In combined segregation and linkage analysis of the complete pedigrees using the first replicate of the simulated data with missing values, we found that the age-of-onset analysis was better at identifying "slope" genes, while the systolic blood pressure analysis was better at identifying "baseline" genes.

Conclusion: Analysis of different trait measures may identify different trait-related genes. When linkage analysis is conducted on multiple trait measures, a linkage signal found for only one measure can represent a true trait locus.
\end{abstract}

\section{Background}

In studies such as the Framingham Heart Study (e.g. [1]) or the Collaborative Study on the Genetics of Alcoholism (COGA) [2], a number of different values related to a disease are measured, and the first step in the analysis may be choosing one measure. The simulated data in Genetic Analysis Workshop 13 (GAW13), for example, offered many measures related to hypertension. Exactly which measure one uses may depend on the analysis method, heritability estimates, and clinical knowledge of the mechanisms behind the disease. Sometimes more than one measure is analyzed and different results are obtained for the different measures, as was see in the GAW11 analyses of the COGA data [3].
Here, we compared two different simulated measures of hypertension in analyses with Monte Carlo Markov chain (MCMC) oligogenic combined segregation and linkage analysis, as implemented in the program Loki [4]. These methods use linkage data on extended pedigrees and estimate the number, location, and effects of loci that contribute to a quantitative trait. We chose two measures of a type that might be collected in a retrospective study: age-ofonset of hypertension $(\mathrm{AOH})$ and systolic blood pressure at the first visit (SBP). We made these choices with knowledge of the generating model. We wanted to determine whether these different measures would localize different trait loci. 


\section{Methods \\ Trait selection}

From the first replicate of the simulated GAW13 data, we selected two traits that were related, but had slightly different genetic characteristics. Both simulated traits we selected, $\mathrm{AOH}$ and SBP at the first visit, are related to hypertension. However, since the generating model divided the trait loci into those that affected "baseline" value and those that affected "slope," we expected that SBP would localize "baseline" genes, while AOH would localize "slope" genes. For SBP we used the SBP value from the first examination in both cohorts. We used the simulated data with missing values. The data set did not contain age-of-onset data as a separate value, but it did contain an indicator of hypertension diagnosis at each visit. We used the age at the earliest visit with a hypertension diagnosis as $\mathrm{AOH}$. Since "baseline" was age 20 at the generating model and the first visit occurred at different ages for different subjects, our SBP measure was not a pure measure of the "baseline." Similarly, since AOH was determined by the crossing of a threshold, $\mathrm{AOH}$ is not a pure measure of "slope." Such "impure" measures may, however, better reflect the measures found in studies of real data.

\section{MCMC segregation and linkage analysis}

To estimate the number, effects, and location of loci contributing to $\mathrm{AOH}$ and SBP, we applied the MCMC segregation and linkage analysis methods described in Heath [4] and Daw et al. [5]. These methods also estimate covariate effects, and the trait model is given by

$$
y=\mu+X \beta+\sum_{i=1}^{k} Q_{i} \alpha_{i}+e,
$$

where $\mu$ is the "reference" trait value, $X$ is the incidence matrix for covariate effects, $\beta$ is the vector of covariate effects, $Q_{i}$ is the incidence matrix for the effects of QTL $i$, $\alpha_{i}$ is the vector of effects for QTL $i, e$ is the normally distributed residual effect, and $k$ is the number of QTL currently estimated $(k \geq 0)$. The MCMC process samples $\mu, \beta$, $\alpha_{i}, i$, and $e$ as well as parameters such as unobserved marker genotypes. All these parameters are sampled from the space of model values consistent with the data observed. Values are sampled proportional to their posterior probability. After the number of sampling iterations is sufficiently large, the sampled values provide an estimate of the posterior probability distribution over the space of possible parameter configurations. We chose a set of covariates that had an effect in the generating model. For comparability, nearly the same covariates were used for both traits. For SBP, the covariates included cigarettes smoked per day (CPD), sex, and age, as reported at the first visit. For $\mathrm{AOH}$, the effects of $\mathrm{CPD}$ and sex were estimated as covariates. AOH covariates are a subset of those for SBP, with only age not used in the case of AOH. The censored trait model described in [5] was used for AOH. This censoring model is essentially a genetic survival analysis with cumulative normal survival curves. Thus, the age information still contributes in both of our analyses.

We conducted a complete genome scan for both traits. We first carried out analyses on both models on all 22 chromosomes using 50,000 iterations, while saving every fifth iteration. On chromosomes with evidence for linkage, we followed up with longer runs. Additionally, for each trait we conducted a longer multi-chromosome analysis including all chromosomes with an L score $>5$. These longer runs were 200,000 to 500,000 iterations in length. All analyses were conducted with the sex-averaged Haldane map provided with the simulated data.

\section{Bayesian "L-score"}

To evaluate evidence for linkage, we considered Bayes factors estimated over 1-cM wide bins along the chromosomes. A Bayes factor is simply the posterior probability divided by the prior probability. In the absence of any data, a Bayesian analysis should have posterior probability equal to the prior probability. Thus, a Bayes factor of 1 indicates that the data contains no information for or against linkage. A Bayes factor $<1$ indicates evidence against linkage, while a Bayes factor $>1$ indicates evidence for linkage. We refer to these Bayes factors for linkage calculated in 1-cM intervals as "L-scores." We used the Haldane map provided with the simulated data.

\section{Results}

In both analyses, there were three regions with L-scores that stood out with values $>50$, while the next largest scores were $<25$. All three regions that were identified in this way contained simulated trait loci (see Table 1). As expected, the largest L-score in the $\mathrm{AOH}$ analysis was at the largest-effect "slope" gene, Gs10. The peak score occurred in a bin adjacent to the one actually containing the locus, so the localization of the gene was within $2 \mathrm{cM}$ of the true location. Also as expected, the largest L-score in the SBP analysis was at the largest effect SBP baseline gene, Gb34, although the peak was some $8 \mathrm{cM}$ away from the true gene location. The second largest "baseline" gene, Gb35, was also identified in the SBP analysis. Of particular interest, the SBP analysis also identified Gs10, although the L-score was somewhat smaller than the $\mathrm{AOH}$ analysis, and the peak value was further from the true gene location than in the $\mathrm{AOH}$ analysis. The $\mathrm{AOH}$ analysis found weak evidence for linkage at Gb34 and no evidence for linkage at Gb35.

Exactly where to draw a threshold for follow-up is not clear. In addition to the three locations with L-scores $>50$, there were several regions with L-scores $>10$ and $<50$. For completeness, we list all regions with L-scores $>10$. For 
Table I: Locations of largest L-scores in single chromosome analysis of AOH and SBPA

\begin{tabular}{|c|c|c|c|c|c|}
\hline \multirow[b]{2}{*}{ QTL } & \multirow[b]{2}{*}{ Location } & \multicolumn{2}{|c|}{$\mathrm{AOH}^{\mathrm{B}}$} & \multicolumn{2}{|c|}{ SBP } \\
\hline & & L-score & Peak & L-score & Peak \\
\hline Gs 10 & $53.59 \mathrm{cM}-\mathrm{chr} .21$ & 94.5 & $52 \mathrm{cM}$ & 76.7 & $49 \mathrm{cM}$ \\
\hline Gb34 & $176.08 \mathrm{cM}-\mathrm{chr} .5$ & 4.8 & $180 \mathrm{cM}$ & 84.4 & $168 \mathrm{cM}$ \\
\hline Gb35 & $85.16 \mathrm{cM}-\mathrm{chr} .13$ & 0.3 & $93 \mathrm{cM}$ & 59.4 & $94 \mathrm{cM}$ \\
\hline
\end{tabular}

AValues and peak locations for largest L-scores. ${ }^{\mathrm{B}} \mathrm{L}$-score values for $\mathrm{AOH}$ analysis at Gb34 and Gb35 included for comparison.

$\mathrm{AOH}$, these L-scores were: $\sim 21$ at $\sim 80 \mathrm{cM}$ on chromosome $5, \sim 22$ at $\sim 95 \mathrm{cM}$ on chromosome $7, \sim 15$ at $45 \mathrm{cM}$ on chromosome 14 , and $\sim 11$ at $\sim 50 \mathrm{cM}$ on chromosome 19. For SBP, these L-scores were: $\sim 14$ at $\sim 220 \mathrm{cM}$ on chromosome 3 and $\sim 11$ at $\sim 95 \mathrm{cM}$ on chromosome 16. Since no trait loci were placed on even-numbered chromosomes, the two signals on even numbered chromosomes represent weak false-positives. It is more difficult to say anything conclusive about the signals on the odd-numbered chromosomes because of the complexity of the generating model: all of the odd numbered chromosomes do, in fact, contain loci that contribute to the simulated hypertension trait. Since more signals in this range of 10 to $~ 20$ are on odd numbered chromosomes, it seems likely that at least some of these are true weak positive signals. The largest effect height gene, Gb1, is at $\sim 80 \mathrm{cM}$ on chromosome 5, while a total of nine different genes are on chromosome 7 , making these signals for AOH likely true weak positives and both of these are over 20. Any threshold value may depend on the investigators tolerance of false-positives, but while the three strong signals (Table 1 ) were all $>50$, the next best signal was $<25$, and the largest score on an even-numbered chromosome was $\sim 15$.

\section{Conclusions}

We find that analysis of $\mathrm{AOH}$ is better at localizing slope genes, while analysis of SBP is better at identifying baseline genes. We noted with interest at GAW 13 that some other groups reported better localization of slope genes with methods that examine value at one time-point rather than "slope." We believe we were able to identify Gs10 in our SBP analysis because most subjects were over the baseline age of 20 at the first visit and the slopes were generally positive, causing the variation in SBP to increase with age. Some alternate generating models were suggested at the GAW 13 meeting under which we would expect our SBP analysis to fail to localize slope loci. Exactly what cut-off should be used for declaring significance with L-scores remains an open question. Our results suggest that analysis of different trait measures can identify different trait loci. Thus, if one has multiple measures, conducts linkage analyses on all of them, and only focuses on those linkage signals replicated in analyses of several measures, one may miss some trait loci.

\section{References}

I. Levy D, DeStefano AL, Larson MG, O'Donnell CJ, Lifton RP, Gavras $\mathrm{H}$, Cupples LA, Myers RH: Evidence for a gene influencing blood pressure on chromosome 17: genome scan linkage results for longitudinal blood pressure phenotypes in subjects from the Framingham Heart Study. Hypertension 2000, 36:477-483.

2. Begleiter H, Reich T, Nurnberger J Jr, Li TK, Conneally PM, Edenberg $H$, Crowe R, Kuperman S, Schuckit M, Bloom F, Hesselbrock V, Porjesz B, Cloninger CR, Rice J, Goate A: Description of the Genetic Analysis Workshop II Collaborative Study on the Genetics of Alcoholism. Genet Epidemiol 1999, 17(suppl I):S25-S30.

3. Almasy L, Borecki IB: Exploring genetic analysis of complex traits through the paradigm of alcohol dependence: summary of GAW I I contributions. Genet Epidemiol I999, I 7(suppI I):SI-S24.

4. Heath SC: Markov chain Monte Carlo segregation and linkage analysis for oligogenic models. Am J Hum Genet 1997, 61:748-760.

5. Daw EW, Heath SC, Wijsman EM: Multipoint oligogenic analysis of age-at-onset data with applications to Alzheimer's disease pedigrees. Am J Hum Genet 1999, 64:839-85I.

\footnotetext{
Publish with BioMed Central and every scientist can read your work free of charge

"BioMed Central will be the most significant development for disseminating the results of biomedical research in our lifetime." Sir Paul Nurse, Cancer Research UK

Your research papers will be:

- available free of charge to the entire biomedical community

- peer reviewed and published immediately upon acceptance

- cited in PubMed and archived on PubMed Central

- yours - you keep the copyright

Submit your manuscript here:

http://www.biomedcentral.com/info/publishing_adv.asp

BioMedcentral
} 